\title{
The Future of Diagnostic Laboratory Testing in Healthcare
}

\author{
Jawahar (Jay) Kalra ${ }^{1,2 *}$, Zoher Rafid Hamed ${ }^{2}$ and Patrick Seitzinger ${ }^{3}$ \\ ${ }^{1}$ Saskatchewan Health Authority, Royal University Hospital, Canada \\ ${ }^{2}$ Department of Pathology and Laboratory Medicine, University of Saskatchewan, Canada \\ ${ }^{3}$ Faculty of Medicine, University of British Columbia, Canada
}

*Corresponding author: Jawahar (Jay) Kalra, Department of Pathology and Lab Medicine, University of Saskatchewan, 103 Hospital Drive, Saskatoon, SK, Canada.

To Cite This Article: Jawahar (Jay) Kalra. The Future of Diagnostic Laboratory Testing in Healthcare. Am J Biomed Sci \& Res. 2019 - 5(2). AJBSR. MS.ID.000883. DOI: 10.34297/AJBSR.2019.05.000883

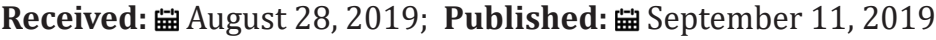

\begin{abstract}
The role of diagnostic laboratory testing in healthcare is evolving. The challenge facing the new era of medicine is the appropriate implementation of new tools and technologies to improve patient care in a cost-effective and sustainable manner. Unprecedented expectations to detect disease earlier and effectively treat all aspects of health and wellbeing create demands for testing which may be premature. Test ordering patterns among physicians are subject to psychological factors such as a desire for certainty and risk aversion, as well as fears of patient dissatisfaction, and litigation. The path towards optimizing the utilization of diagnostic laboratory tests must include strategies to minimize non-contributory testing. Appropriate application and sound clinical reasoning are essential to mitigating overdiagnosis, exponential costs on the healthcare system and unnecessary suffering on the part of the patient. Reducing non-contributory laboratory testing practices would allow for the reallocation of resources toward the protection of imperative practices and the advancement of strategies in preventative and individualized medicine. With thoughtful deliberation and constructive conversations among all stakeholders these challenges, pressures and disruptions have the potential to create innovative strategies to optimize the use of diagnostic laboratory testing in healthcare.
\end{abstract}

Keywords: Laboratory Testing; Diagnostic Medicine; Preventative Medicine; Implementation; Overdiagnosis; Sustainable Healthcare

Abbreviations: TSH: Thyroid Stimulating Hormone; T3: Triiodothyronine; T4: Thyroxine

\section{Introduction}

The challenge of medicine, including diagnostic laboratory medicine, is to implement new tools and technologies in a costeffective and sustainable manner to improve patient care. In the 1960 's researchers realized that the potential infinite demands of healthcare could not be accommodated by the finite resources available [1]. The evolving trends and circumstances of modern medicine may be considered an 'innovative disruption' to the practice of laboratory medicine [2]. Such evolving circumstances include but are not limited to technological advances, demographic changes, complexity of care, and increases in the expectations of well-informed patients [3]. With careful, thoughtful, and deliberate guidance, this disruption can lead to the betterment of healthcare and serve the interests of the patient. Achieving this balance is challenging and will require a paradigm shift in the perspectives and practices of laboratory testing to keep costs from increasing exponentially and to mitigate potential consequences.

\section{Contributing Factors}

Demand plays an important role in the practice of ordering laboratory tests. Both direct and indirect pressures on physicians to order a broad range of tests, and in many cases repeat these tests, arise from multiple sources including researchers, manufacturers, government, and often from patients themselves. In today's medical culture, ordering tests and procedures is often interpreted by patients as synonymous with sign of a thorough doctor [4] and due diligence. These expectations create demands for testing which may in some circumstances be premature.

Test ordering patterns are further influenced by physician realities. Factors such as a desire for certainty and a natural behavioral tendency for risk aversion influence the physician's decision on the necessity and cost-benefit balance of laboratory tests. Physicians are weary to omit any test due to fears of missing a diagnosis, patient dissatisfaction, and litigation. While 
the magnitude of these factors is unprecedented, the physician's core dilemma of balancing autonomy of the patient and avoiding maleficence through overdiagnosis remains unchanged. As societies expectations of physicians to detect disease earlier, cure disease and treat all aspects of health and wellbeing continue to grow exponentially, as will the implications that these expectations have on the efficiency, quality, and justice of the healthcare system.

\section{Awareness of the Problem}

The awareness of the ubiquity of over testing is pervasiveness in healthcare throughout North America. Despite the dilemmas presently associated with the choice of laboratory tests, everyone involved in medical care today is aware that there are situations in which inappropriate (excessive or unnecessary) laboratory testing occurs [5]. Approximately 25\% of Canadians report that their physician ordered a test that they considered unnecessary [4]. Furthermore, about one third did not follow their physician's advice and did not get the test performed [4]. Despite differences in funding structure, no considerable differences were found in the use of laboratory testing in Canada and the United States [6]. In 2014, more than $70 \%$ of American physicians reported that they believe the average doctor orders at least one unnecessary test or treatment per week [4].

The perceived normality and status quo of these practices leads to further propagation of demand. In the past decade, the volume of laboratory tests being performed has been increasing at a rate of 6-8\% per year [7], while per capita laboratory costs have increased 8-34\% over 5 years [8]. With an aging population of baby boomers, this increase in tests and treatments being administered without a clear corresponding improvement in health is of considerable concern [4].

\section{Strategies Currently in Place}

Realizing the dangers of inappropriate and unnecessary testing, various initiatives have been established to mitigate the problem. Efforts such as clinical auditing [9] and administrative interventions [10] have shown limited practicability with questionable sustainability. In our previous work, we have shown the utility of TSH alone during the screening of thyroid disorders, thereby eliminating unnecessary T3 and T4 tests in diagnosis [11] and optimizing treatment [12]. This has been tremendously effective in optimizing testing of specific thyroid markers. However, to create a widespread impact on laboratory testing in all areas of medicine, a change in perspective is necessary. Examples of efforts to change perspectives on laboratory testing include Choosing Wisely, which aims to facilitate conversations between patients and physicians around choosing only care that is necessary and avoiding harm [4]. On a more administrative level, Lean methodology has been applied to healthcare with the objective to reduce waste, improve quality and create a supportive network through management strategies [13].

As the accessibility of laboratory results and health related data through online portals is increasing, conversations about the meaning and individual context of test results and effects on treatment are becoming increasingly important [14]. Despite the influence that expectation and pressures may have, laboratory utilization is the responsibility of the medical profession. We must accept that we are responsible for our present situation and lead the way in rectifying the current trajectory of laboratory medicine.

\section{Minimizing Non-Contributory Medical Practices}

The purpose of laboratory medicine is to provide evidence of the current state of a patient's health to inform the prevention, treatment or management of an existing or impending disease or condition [15]. Despite previous efforts, the unnecessary maximization and overutilization of tests continues in areas that are not fruitful. This has lasting maladaptive consequences on the allocation of laboratory resources. As doctors are pressured to order more tests, increases in testing practices continues to have an associated increase in costs on the medical system. As costs continue to rise, funding agencies are applying pressure on doctors to decrease the magnitude of test being performed. These pressures will undoubtedly continue to have significant impacts on the clinical practice of ordering laboratory tests.

Although the accuracy of laboratory tests continues to progress, false positives and false negatives still occur. When careful application and reflection is overlooked in the practice of laboratory testing, overdiagnosis is likely to take place. Even with the best of intentions, over-testing is not in the best interest of the patient and may lead to overdiagnosis, unnecessary costs on the healthcare system and suffering on the part of the patient [4]. As stated by Dr. Martin "If over-testing and overtreatment were a disease, we would declare it an international epidemic" [4].

When ordering any test, the physician should pre-emptively consider the implications that the result will have on treatment and communicate these considerations to the patient. When multiple tests are ordered without adequate clinical justification, each unnecessary test adds data pollution, and reduces the signal to noise ratio [16] and may produce results that unnecessarily complicate the clinical situation. Ensuring that sound clinical reasoning is at the core of every decision to order each laboratory test would likely alleviate a considerable portion of the roughly $30 \%$ of laboratory tests ordered that is currently attributed to repeat testing [16]. This would enable more allocation of resources to sustainable strategies that contribute to patient care.

\section{Maximizing Imperative Medical Practices}

Essential components of patient care must be maintained and strengthened, regardless of pressures, political agendas and funding restrictions. Despite the widespread tendency to over test, the opposite also exists - situations in which appropriate laboratory testing does not occur [5]. As new laboratory test technology moves us toward a preventative approach to maintaining health, rather than being limited to the reactive disease management approach of the past, the definition of essential components of patient care is evolving. Examples of such transitions toward a preventative approach in laboratory medicine is seen in diseases such as leukemia, breast cancer and heart disease with new technology including genome, proteome and cellulome guided disease management [17]. The role of laboratory medicine in the new era of 
clinical care continues to expand to encompass more screening and early diagnosis as well as determining disease susceptibility and confirming a state of well-being. Studies have found that these new applications represent more than $50 \%$ of the current laboratory workload [18]. While this may seem unnecessary at present but will have lasting benefits for the medicine of tomorrow.

At a population level, disease susceptibility studies inform what diseases are unlikely and need not be tested for at as regular of intervals in certain groups, thereby avoiding unnecessary tests in these low risk segments of the population. In at-risk segments of the population, it will inform what risk factors are to be avoided and the interval at which particularly likely diseases should be screened for. This information allows for prevention at the primordial and primary levels and allows for the safest most effective treatment. If prevention cannot be achieved, the appropriate medical judgement to take the necessary steps to ensure a proper diagnosis is made at the first manifestation of disease. Tailored treatments can then be delivered to the individual, resulting in an overall increase in the efficiency of the medical system. Appropriate application is key to the implementation of early diagnoses and aggressive treatments as well as the use of expensive tests and procedures [4]. These standards of practice will support physicians in providing quality medical care while also acting as a cost-conscious healthcare participant.

At an individual level, therapeutic decisions will rely less on comparisons of the concentration of single molecular markers to reference values of a healthy, yet not necessarily relatable, subset of the population. Instead, therapeutic decisions will be informed more by changes of the patient's individualized profile of markers [19]. This will allow for comparisons between the patient's status and physiological circumstances to the expected response of a particular patient to a particular treatment. This movement has the potential to produce more accurate diagnoses, safer tailored treatments leading to faster and more effective recovery and decrease costs in the long run [19]. As new technology brings about this era of personalized medicine, it will become increasingly important to strengthen collaborations among clinicians with pathologists and medical laboratories to ensure accurate implementation and interpretation of laboratory tests.

\section{Conclusion}

The path towards optimizing the utilization of diagnostic laboratory tests in this new era must include strategies to reduce the current unnecessary and non-contributory testing as well as strategies to ensure the protection and advancement of essential services. Reducing non-contributory laboratory testing practices will allow us to build and strengthen strategies in disease prevention and early diagnosis. This transition in the practice of laboratory testing will require a change in the culture of medicine as well as communication between patients, physicians, administrators, funding agencies, lawmakers, and manufacturers. Only through a common understanding among each of these stakeholders can we achieve progress in optimizing the health of the population in a cost-effective and sustainable manner.

\section{Conflict of Interest}

There are no conflicts of interest to declare.

\section{References}

1. Emson HE (1965) The Growth of a Laboratory. Can Med Assoc J 93: 211218.

2. Christensen CM, Raynor M, Mcdonald R (2015) What Is Disruptive Innovation? Harvard Business Review p. 1-11.

3. Jorgensen PE (2019) What is happening to laboratory medicine in Denmark? Clin Chem Lab Med 57(3): 349-352.

4. Martin D (2017) Better now: six big ideas to improve health care for all Canadians, Allen Lane, Toronto, Ontario, Canada.

5. Epstein AM, McNeil BJ (1987) Variations in ambulatory test use: what do they mean? Med Clin North Am 71(4): 705-717.

6. Katz SJ, Mcmahon LF, Manning WG (1996) Comparing the Use of Diagnostic Tests in Canadian and US Hospitals. Med Care 34(2): 117125 .

7. Naugler C (2014) A perspective on laboratory utilization management from Canada. Clin Chim Acta 427: 142-144.

8. Ndegwa S (2011) Funding of Laboratory Testing in Canada [Environmental Scan, Issue 19]. Can Agency Drugs Technol Heal (19): $1-5$.

9. Huang Y, Don Wauchope AC, Grey VL, Mansour M, Brill H, et al. (2012) Improving serological test ordering patterns for the diagnosis of celiac disease through clinical laboratory audit of practice. Clin Biochem 45(6): 455-459.

10. Van Walraven C, Goel V, Chan B (1998) Effect of population-based interventions on laboratory utilization: A time-series analysis. J Am Med Assoc 280(23): 2028-2033.

11. Kalra J, Laxdal VA, Walker SD, Massey KL (1987) Evaluation and assessment of high sensitivity thyrotropin methods as an index for thyroid function. Clin Biochem 20(5): 307-312.

12. Kalra J, Hart IR (1987) Value of free thyroxine (FT4), free triiodothyronine (FT3), and sensitive thyrotropin (TSH) assay in the assessment of optimal thyroxine therapy. Clinical biochemistry 20(4): 265-267.

13. Kinsman L, Rotter T, Stevenson K, Bath B, Goodridge D, et al. (2014) "The Largest Lean Transformation in the World": The Implementation and Evaluation of Lean in Saskatchewan Healthcare. Healthc Q 17(2): 29-32.

14. Witteman HO, Zikmund-Fisher BJ (2018) Communicating laboratory results to patients and families. Clin Chem Lab Med 57(3): 359-364.

15. Holladay EB (2010) Bring on the future of lab medicine. MLO Med Lab Obs 42(7): 62.

16. Van Walraven C, Raymond M (2003) Population-based Study of Repeat Laboratory Testing. Clin Chem 49(12): 1997-2005.

17. Kershaw J (2008) Sustaining the future of lab medicine. MLO Med Lab Obs 40(8): 52.

18. Ceriotti $F$ (2019) Is there a classical role for the clinical laboratory in digital health? Clin Chem Lab Med 57(3): 353-358.

19. Žitnik IP, Zerne D, Mancini I, Simi L, Pazzagli M, et al. (2018) Personalized laboratory medicine: A patient-centered future approach. Clin Chem Lab Med 56(12): 1981-1991. 